\title{
Awareness of Breathing: The Structure of Language Descriptors of Respiratory Sensations
}

\author{
Sibylle Petersen and Bernhard Orth \\ University of Hamburg
}

\author{
Thomas Ritz \\ Southern Methodist University
}

\begin{abstract}
Objective: Recent research suggests that dyspnea is not a single sensation but a multidimensional construct reflected in different verbal descriptors that can provide useful diagnostic information. In this study superordinated clusters of dyspnea were investigated in combination with a dimensional approach. Design: We examined the use of 20 respiratory symptom descriptors by healthy volunteers who completed a protocol of seven experimental conditions: Quiet breathing, breath holding, paced breathing, climbing stairs, resistive load breathing, voluntary hyperinflation, and voluntary hyperventilation. Main Outcome Measures: We analyzed the ratings of these descriptors with multidimensional scaling (MDS) and cluster analysis. Results and Conclusion: While similarities with prior studies were found on a lower fusion level, we were able to demonstrate the usefulness of interpreting higher fusion levels with four clusters related to work of breathing, coordination, suffocation, and struggling for air, merging into two superordinated clusters, effort and air hunger that are compatible with widely accepted primary components of dyspnea. MDS results also suggested that future studies should consider further breathing sensations related to cognitive control of breathing.
\end{abstract}

Keywords: dyspnea, symptom perception, respiratory sensation, verbal descriptors

The perception of breathlessness is an integral part of the symptomatic manifestation of psychological disorders and organic diseases, such as panic disorder, asthma, chronic obstructive pulmonary disease, or cardiopulmonary diseases (Manning and Schwartzstein, 1995; Meuret, White, Ritz, Roth, Hofmann, \& Brown, 2006). Research suggests that dyspnea is not a single sensation, but a multidimensional construct that is reflected in language in the use of different descriptors of breathlessness (e.g. Harver, Mahler, Schwartzstein, \& Baird, 2000; Mahler, Harver, Lentine, Scott, Beck, \& Schwartzstein, 1996; Simon, Schwartzstein, Weiss, Lahive, Fencl, Teghtsoonian, 1989). Three (Perna, Caldirola, Namia, Cucchi, Vanni, \& Bellodi, 2004) up to 12 (Elliott, Adams, Cockcroft, MacRae, Murphy, \& Guz, 1991) types of dyspnea have been suggested, with a limited overlap between studies of healthy and clinical populations (e.g. Harver et al., 2000; Simon et al., 1989).

While studies on verbal descriptors are variable, physiological analysis suggests that a smaller number of dyspnea components can be distinguished more clearly, a sense of effort, the feeling of hunger for air, chest tightness, and problems not getting enough air (e.g., American Thoracic Society, 1999; Binks, Moosavi, Banzett, \& Schwartzstein, 2002; Lansing, Im, Thwing, Legedza, \& Banzett, 2000). We suspected that methods used in prior studies of lan-

Sibylle Petersen and Bernhard Orth, Department of Psychology, University of Hamburg, Hamburg, Germany; Thomas Ritz, Department of Psychology, Southern Methodist University, Dallas, Texas.

This work was partly funded by grants from the German Research Society (DFG Ri 957/3-1).

Correspondence concerning this article should be addressed to Sibylle Petersen, Department of Psychology, University of Hamburg, Von-Melle-Park 5, 20146, Hamburg, Germany. E-mail:sibylle.petersen@uni-hamburg.de guage descriptors may have overestimated both the number of relevant clusters and similarities between healthy and clinical populations. In hierarchical cluster methods, small clusters with one or two items are easier to replicate than larger clusters. Because healthy individuals are less frequently exposed to dyspneic sensations they may actually have less complex concepts of dyspnea. Therefore, by using standard decision rules we sought to determine the number of clusters that could be reasonably discriminated by healthy individuals. Rather than stopping at an earlier fusion level in cluster analysis, as earlier studies did, solutions on a higher level of cluster fusion may be more appropriate. We suspected that if such a solution would be justified, it would converge onto proposed primary components of dyspnea. In addition, by using multidimensional scaling (MDS) we explored whether a dimensional interpretation could offer a more plausible structure compared to an interpretation in terms of discrete clusters of respiratory sensations.

\section{Method}

\section{Participants}

Participants were 14 individuals (12 women, mean age 29.2 years, range 21-41 years), who reported being non-smokers and having no cardiovascular or respiratory disease. They received course credits for their participation. Participants gave consent initially and were then debriefed in detail after the experimental session.

\section{Instruments}

A list of 20 descriptors of respiratory sensations composed by Simon et al. (1989) was translated and adapted to German language (Appendix). Because there is only one counterpart for 
"suffocating" and "smothering" in German language, only one descriptor referring to this sensation was included. As common German expressions we included "I pant for air" and "I am running out of air". A descriptor list closer to the English original would have allowed a comparison between cultures, however, this would have lowered the external validity of such a list for our German sample and our focus was rather on superordinated pattern of sensations than on such an intercultural approach. Each descriptor was rated on a 11 -point scale $(0=$ not at all, $10=$ very strong $)$. Participants also reported on their age, body weight, and medical history in an ad-hoc questionnaire.

\section{Design and Procedure}

The participants completed a protocol of seven conditions varying in intensity and quality of the challenge to the respiratory system: (1) Quiet sitting: Participants were instructed to sit quietly with their eyes open for $2 \mathrm{~min}$. They were then asked to rate their respiratory sensations during the task. (2) Breath holding: The participants were told to hold their breath for as long as possible. They were then asked to rate how breathless they felt at the moment they started to breathe again. (3) Paced breathing: Participants adapted their respiration rate to a pacing tone presented using a tape recorder. Three different speeds were presented, each for 3 min: 8, 13, and 18 breaths/min. Following the third and fastest speed, the participants rated their respiratory sensations for this final task period. (4) Climbing stairs: Participants climbed a set of stairs to the $7^{\text {th }}$ floor. The instruction was to climb at a steady pace. At arrival on the $7^{\text {th }}$ floor they rated their current respiratory sensations. (5) Added resistive load breathing: Participants breathed through a mouthpiece and tube $(80 \mathrm{~cm}, 85 \mathrm{ml}$ deadspace $)$ for $2 \mathrm{~min}$ with the nose occluded. An added resistive load $(0.75$ $\mathrm{kPa} / \mathrm{l} / \mathrm{s}$ ) was attached to the distal end of the tube. The experimenter used the digital display of a capnometer to monitor partial pressure of carbon dioxide in the exhaled air (end-tidal $\mathrm{pCO}_{2}$ ) which had to remain below $45 \mathrm{mmHg}$. Following the task, participants rated their sensations during the task. (6) Voluntary hyperinflation: Participants were instructed to breathe in from resting end-expiratory level to total lung capacity, then to breathe out approximately one-third of the way, and then to continue breathing on that level for $1 \mathrm{~min}$. Afterwards, they rated their respiratory sensations during the task. (7) Voluntary hyperventilation: Participants were asked to follow the pacing tone from an audio tape at 18 breaths/min and at the same time breathe with a high tidal volume to reach an end-tidal $\mathrm{pCO}_{2}$ of $20 \mathrm{mmHg}$ (feedback given by the digital display of the capnometer). They were then asked to hold this $\mathrm{pCO}_{2}$ level for $1 \mathrm{~min}$. Following the task they were asked to rate their current respiratory sensations.

The experimental tasks were administered in random order across participants in individual laboratory sessions. Variable breaks between the exercises were given, with a minimum of 10 min after both voluntary hyperventilation and climbing stairs.

\section{Data Analysis}

Before combining judgments to mean ratings for each item per condition, we computed interrater reliabilities (Inter Class Correlations (ICC), Shrout \& Fleiss, 1979) and excluded descriptors with an $I C C<.75$. Our analysis of the relationships between items was based of the average within-subject structure of each item regarding the seven conditions. Therefore it was important to exclude items for which only low agreement was obtained.

We used two different methods of analysis, cluster analysis using the Single Linkage fusion algorithm and squared Euclidian distances and MDS using squared Euclidian distances. Both methods analyze the relationship between objects on the basis of their mutual similarities or distances with regard to various attributes. In our analysis the seven conditions served as "attributes" and distances between items were computed with reference to the ratings of all seven conditions simultaneously. The decision on the number of clusters to interpret involved two aspects: (i) the homogeneity of the cluster compared to the heterogeneity of the whole group, and (ii) differentiation between clusters (Jardine \& Sibson, 1971). Plotting a heterogeneity coefficient as a function of the number of clusters, similar to a scree-plot in factor analysis, it is possible to decide if an earlier fusion level with more and smaller clusters leads to a reasonable increase of homogeneity within the clusters. The second question concerning the differentiation between clusters can be answered by computing a linear discriminant analysis, using the groups of items identified by cluster analysis as input (Méndez, Hödar, Vulpe, González, \& Cambiazo, 2002).

In a broader sense MDS can be regarded as a theory of mental stimulus representation (Torgerson, 1958). The scaling algorithm attempts to find a minimum of dimensions that best satisfy the mutual distances between items. Deciding which number of dimensions to choose, the interpretability of the dimensions was taken into account as well as Kruskal's stress formula 1 as a "badness of fit" measure (Kruskal \& Wish, 1994). While MDS reveals continuous dimensions that underlie the similarities of objects, cluster analysis displays the degree of similarities between objects in a more particular fashion and combines them into distinct subgroups. Both methods could theoretically be applied to ratings of one person only. Therefore the number of participants was less important, but the number of items and the agreement between participants regarding these items. A heuristic rule of Kruskal and Wish (1994) states that $I-1>4 R$, where $I$ is the number of stimuli (sensation items) and $R$ the number of dimensions.

A joint representation of MDS and cluster results indicates to what degree these two methods lead to congruent findings (Kruskal \& Wish, 1994). The cluster-solution is thereby embedded in the MDS configuration by drawing ellipses around the items of each cluster. A perfect joint representation can only be obtained if clusters can be encircled in such a way that their contours form convex surfaces only and not concave ones (e.g. by loops being forced to slide around one item not belonging to a cluster) and if cluster surfaces do not overlap.

\section{Results}

\section{Cluster Analysis}

Five items with an $I C C<.75$ were identified and excluded: "I feel that I am smothering" (ICC $=.53)$, "My chest is constricted" $(I C C=.68)$, "I feel that my breath stops" (ICC $=-.07)$, "My breathing is shallow" (ICC $=.65)$, and "I am running out of air" $(I C C=.44)$. Cluster analysis identified four clusters which we interpreted as: 1) coordination of breathing, 2) work of breathing, 
3) feelings of suffocation, 4) struggling for air (Figure 1). These clusters merge to two superordinate clusters, interpreted as effort (cluster 1,2) and air hunger (cluster 3, 4). According to the visual inspection of the dendrogram, a two-cluster-solution would be possible just as well as an eight-cluster-solution including outlier items interpreted as clusters as done in prior research. A scree-plot (not displayed here) showed no further substantial decrease in heterogeneity after a four-cluster-solution. Thus, earlier fusion levels do not lead to more homogeneous clusters. A linear discriminant analysis using the groups recovered by cluster analysis as input showed that the four clusters were statistically separated (Wilks' lambda $=.16 ; \operatorname{chi}^{2}(5)=12.14 ; p=.03$ ).

The Single Linkage algorithm is especially useful for discovering outliers (Jardine \& Sibson, 1971). Item 14 (breathing more) joined its cluster very late and item 6 (out of breath) did not join any cluster at all. Item 19 (concentration) also constituted an outlier. It was the only item that described cognitive effort in breathing. After excluding these items, the results of the Single Linkage algorithm were confirmed by the non-hierarchical k-means method which can be regarded as a sign of a stable solution (results can be obtained by request).

\section{Interaction of Clusters and Experimental Tasks}

A one-way repeated measure ANOVA of the experimental conditions showed significant differences between quiet sitting and the six tasks that induced respiratory sensation in different ways, $F(6,66)=12.40, p<.001, \eta^{2}=.53$. Quiet sitting was rated less intense than the other tasks, post hoc tests (Least Significant Difference) $p<.02$, for five conditions, except breath holding, $p=.30$. The two tasks with the greatest overall intensity were stair climbing and hyperinflation. To display the intensity ratings for each condition separately for each cluster in each condition we computed standardized $t$-values for each experimental task for every cluster. These $t$-values were computed as the difference of the mean condition ratings by one cluster with the mean condition ratings by all clusters divided by the standard deviation of the condition across all clusters (Wishart, 1982). Values near zero indicate that a task contributed little to the formation of this cluster. Negative values indicate that this task has characteristics that are opposite to the cluster characteristics and positive values indicate that the task characteristics are very close to the characteristics of the cluster. Figure 2 displays the interactions between clusters and situations. Hyperinflation shows higher $t$-values for the clusters coordination and work, whereas the $t$-values for stair climbing are higher in struggling for air and lower in suffocation and coordination. Stair climbing and hyperinflation did not differ in their mean dyspnea rating. Thus, clusters seem to be determined primarily by the quality and not by the intensity of dyspnea.

\section{Dimensions of Dyspnea Identified in Multidimensional Scaling Analysis}

An ordinal MDS analysis identified three dimensions interpreted as, 1) need, 2) effort, and 3) attempt of voluntary control. Again, items with an $I C C<.75$ were excluded. A stress value of .018 (Kruskal's stress formula 1) indicated an excellent fit for the dimensionality of the data. Figure 3 shows one of the two twodimensional maps in which the three dimensional configuration was decomposed for the sake of clarity. It displays the dimensions need and attempt of voluntary control. The dimension need ranged between items 13 (tight) and 6 (out of breath). Effort (not displayed here) ranged between items 14 (rapid) and 9 (work) and the dimension, attempt for voluntary control ranged between the items 19 (concentration), and 6 (out of breath). The outlier status of item 14 (rapid), item 6 (out of breath), and item 19 (concentration) which we already observed in cluster analysis, was confirmed in MDS and could be described in more detail regarding the identified dimensions. The first two items described a form of dyspnea

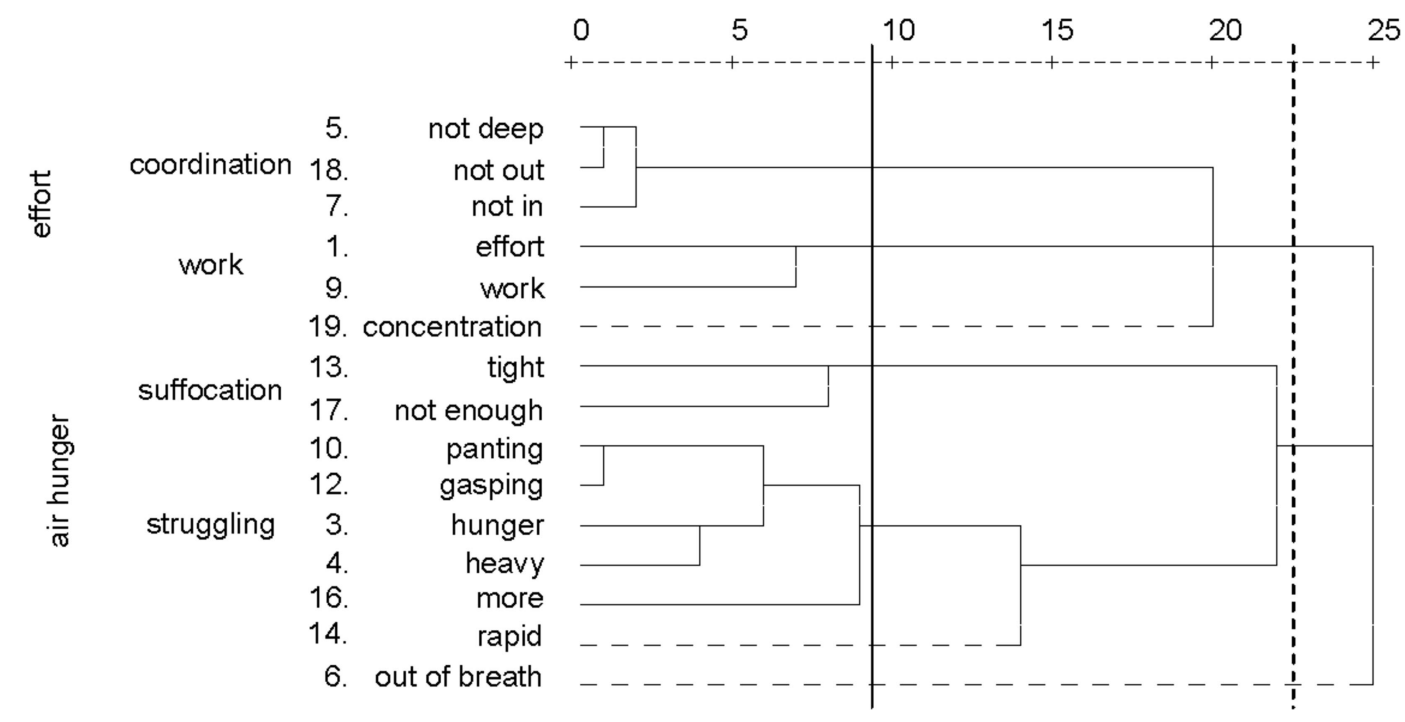

Figure 1. Single-linkage dendrogram resulting from hierarchical cluster analysis using squared Euclidian distances. Vertical lines are indicating the fusion levels of the four cluster solution and the superordinated two clusters solution (dashed line). Horizontal dashed lines are indicating outliers. 


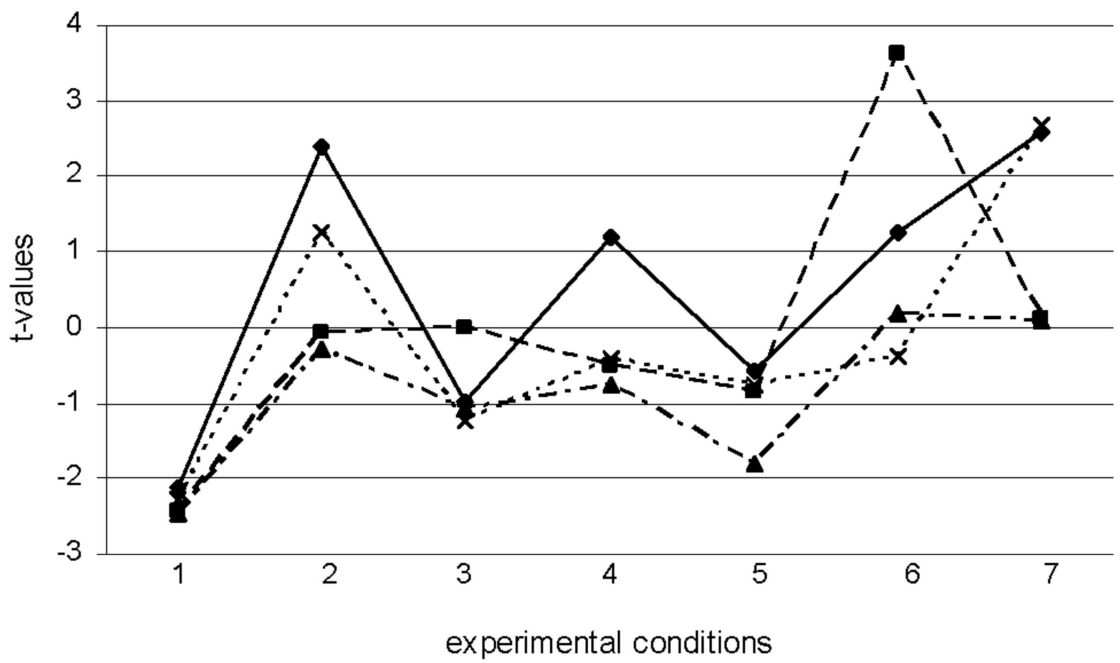

$\longrightarrow$ work $-\rightarrow--$ struggling $-\cdot \star--$ suffocation $\cdots \cdot \times \cdot \cdot$ coordination

Figure 2. T-values of the seven experimental conditions for each of the four clusters: 1) quiet sitting, 2) paced breathing, 3) breath holding, 4) added resistive load breathing, 5) hyperventilation, 6) stair climbing, 7) hyperinflation.

low in need as well as in attempt of voluntary control and effort. In contrast, item 19 ranged from high in attempt of voluntary control over neutral in need to moderately high in effort.

Between an alternative two-dimensional (stress .07) and this three dimensional MDS solution (stress .018) was a substantial decrease in stress, justifying this more complex model. The joint representations (one displayed in Figure 3) showed a good fit between MDS and cluster-solution. The clusters could be embed- ded in the configurations as clearly separated ellipses which suggested internal validity for the uncovered structure.

\section{Discussion}

In this study we explored the structure of the language descriptors of respiratory sensations in healthy individuals. Rather than stopping at a lower fusion level and reporting small but many

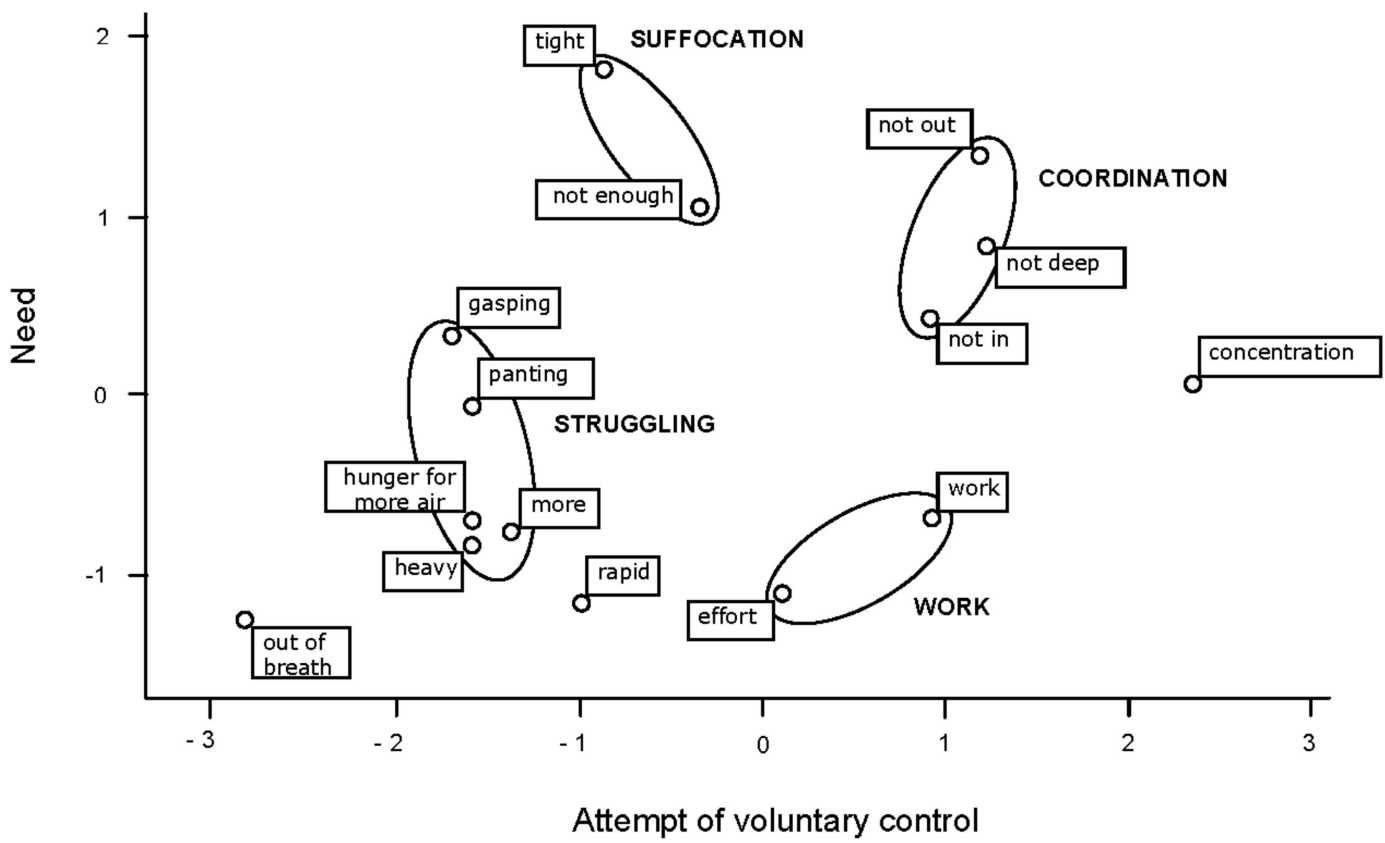

Figure 3. Dimensions need and attempt of voluntary control of the three-dimensional MDS configuration using squared Euclidian distances. 
clusters, we decided to interpret a solution with four basic clusters that joined into two primary elements of dyspnea effort and air hunger, which is in line with results of physiological analysis (e.g. Binks et al., 2002). We demonstrated sensitivity of these clusters to a range of respiratory challenge tasks depending on quality but not on intensity of induced dyspnea. Interpreting advanced levels of fusion could generate additional information regarding processes that lead from isolated respiratory sensations to more complex forms of dyspnea. Respiratory and cardiopulmonary diseases seem to be associated with a unique set of respiratory sensation clusters (e.g. Manning \& Schwartzstein 1995), but more research is needed on the relationship between these clusters and the way in which they converge to primary elements of dyspnea in disease. Future research should explore whether the specific fusion patterns at higher fusion levels differ between healthy and diseased individuals, as well as between different diseases.

A focus on these basic components of respiratory sensation may be more valid for diagnostic purposes. In order to access patients' cognitive representations of symptomatic states, multiple descriptors rather than isolated sensations as suggested by previous cluster solutions may be needed. The coordination cluster for example is constituted by three items related to problems involving breathing in and/or out fully. Healthy individuals are certainly able to differentiate sensations of inhalation and exhalation, but with respect to our respiratory challenge tasks requiring attention to coordination (hyperinflation, i.e. breathing more in than out; and paced breathing, i.e. conscious coordination of inhalation and exhalation) a joint cluster seemed more reasonable. Problems with coordination of breathing related to speech have been observed in clinical conditions such as asthma and chronic obstructive pulmonary (Lee, Friesen, Lambert, \& Loudon, 1998; Skevington, Pilaar, Routh, \& Macleod, 1997). Items grouped in a coordination of breathing cluster could be specifically sensitive to this aspect of dyspnea.

Our findings with MDS confirmed the integrity of the identified clusters and also emphasize attempt of voluntary control as additional cognitive dimension of dyspnea. Originally, Comroe (1966) identified a dimension "awareness of increased ventilation" in addition to other dimensions of respiratory sensations that were conceptualized more as purely sensory or behavioral. Beyond that, cognitive effort in breathing has largely been ignored. The perceived requirement of controlling breathing actively may constitute an alarm signal similar to increased effort or the need to breathe.

A limitation of our study may have been that the selection of our tasks could have partly determined the outcome of the analysis. The limited role of items of the suffocation subdomain may be suggestive of that. However, similar findings were reported by Simon et al. (1989) who presented their healthy participants with a battery of eight tasks, including $\mathrm{CO}_{2}$ inhalation and resistive load challenge. A larger range of different respiratory challenges, including induced respiratory stimulation or bronchoconstriction with pharmacological agents, may be ecologically more valid elicitors of respiratory sensations for patients (Abelson, Nesse, Weg, \& Curtis, 1996). Also the population from which we sampled was a mostly young, healthy student population, thus limiting the generalizability of findings.

Bodily sensations such as dyspnea can only be described sufficiently by the person who experiences it. However, confronted with such a task during an acute illness, patients are often unprepared to provide specific details about their symptoms. A questionnaire detailing aspects of breathlessness could provide patients with guidance to describe and quantify their perceptions more precisely and could help physicians to provide the best diagnosis and treatment. Also, a certain proportion of patients with lung disease have been found to only poorly perceive their internal states, thus making them susceptible to fatal or near-fatal complications (Banzett, Dempsey, O’Donnell, \& Wamboldt, 2000). It has been suggested that a dyspnea questionnaire in combination with a methacholine provocation test provoking airway obstruction could serve to identify such "poor perceivers" (De Peuter, Van Diest, Lemaigre, Verleden, Demedts, \& Van den Bergh, 2004; Julius, Davenport, \& Davenport, 2002). Greater clarity with regard to basic physiologically relevant components of dyspnea would inform this approach. Future studies must also determine the role of emotional states (Rietveld \& Prins, 1998) or traits (Chen, Hermann, Rodgers, Oliver-Welker, \& Strunk, 2006) in affecting basic aspects of respiratory sensation.

\section{References}

Abelson, J. L., Nesse, R. M., Weg, J. G., \& Curtis, G. C. (1996). Respiratory psychophysiology and anxiety: Cognitive intervention in the doxapram model of panic. Psychosomatic Medicine, 58, 302-313.

American Thoracic Society. (1999). Dyspnea. Mechanisms, assessment, and management: A consensus statement. American Journal of Respiratory and Critical Care Medicine, 159, 321-340.

Banzett, R. B., Dempsey, J. A., O'Donnell, D. E., \& Wamboldt, M. Z. (2000). Symptom perception and respiratory sensation in asthma. American Journal of Respiratory and Critical Care Medicine, 162, 1178 1182.

Binks, A. P., Moosavi, S. H., Banzett, R. B., \& Schwartzstein, R. M. (2002). "Tightness" sensation of asthma does not arise from the work of breathing. American Journal of Respiratory and Critical Care Medicine, $165,78-82$.

Chen, E., Hermann, C., Rodgers, D., Oliver-Welker, T., \& Strunk, R. (2006). Symptom perception in childhood asthma: The role of anxiety and asthma severity. Health Psychology, 25, 389-395.

Comroe, J. (1966). Summing up. In: J. B. L. Howell, \& E. J. M. Campbell (Eds.), Breathlessness (pp. 233-238). London: Blackwell Scientific Publications.

De Peuter, S., Van Diest, I., Lemaigre, V., Verleden, G., Demedts, M., \& Van den Bergh, O. (2004). Dyspnea: The role of psychological processes. Clinical Psychology Review, 24, 557-581.

Elliott, M. W., Adams, L., Cockcroft, A., MacRae, K. D., Murphy, K., \& Guz, A. (1991). The language of breathlessness. Use of verbal descriptors by patients with cardiopulmonary disease. American Review of Respiratory Disease, 144, 826-832.

Harver, A., Mahler, D. A., Schwartzstein, R. M., \& Baird, J. C. (2000). Descriptors of breathlessness in healthy individuals. Distinct and separable constructs. Chest, 118, 679-690.

Jardine, N., \& Sibson, R. (1971). Mathematical taxonomy. New York: Wiley.

Julius, S. M., Davenport, K. L., Davenport, P. W. (2002). Perception of intrinsic and extrinsic respiratory loads in children with life-threatening asthma. Pediatric pulmonology, 34, 425-33.

Kruskal, J. B., \& Wish, M. (1994). Multidimensional scaling. Newbury Park, CA: Sage 
Lansing, R. W., Im, B. S-H., Thwing, J. I., Legedza, A. T. R., \& Banzett, R. B. (2000). The perception of respiratory work and effort can be independent of the perception of air hunger. American Journal of Respiratory and Critical Care Medicine, 162, 1690-1696.

Lee, L., Friesen, M., Lambert, I. R., \& Loudon, R. G. (1998). Evaluation of dyspnea during physical and speech activities in patients with pulmonary disease. Chest, 113, 625-632.

Mahler, D. A., Harver, A., Lentine, T., Scott, J. A., Beck, K., \& Schwartzstein, R. M. (1996). Descriptors of breathlessness in cardiorespiratory diseases. American Journal of Respiratory and Critical Care Medicine, 154, 1357-1363.

Manning, H. L., \& Schwartzstein, R. M. (1995). Mechanisms of diseases: Pathophysiology of dyspnea. The New England Journal of Medicine, $333,1547-1553$.

Méndez, M. A., Hödar, C., Vulpe, C., González, M., \& Cambiazo, V. (2002). Discriminant analysis to evaluate clustering of gene expression data. FEBS Letters, 522, 24-28.

Meuret, A. E., White, K. S., Ritz, T., Roth, W. T., Hofmann, S. G., \& Brown, T. A. (2006). Panic attack symptom dimensions and their rela- tionship to illness characteristics in panic disorder. Journal of Psychiatric Research, 40, 520-527.

Perna, G., Caldirola, D., Namia, C., Cucchi, M., Vanni, G., \& Bellodi, L. (2004). Language of dyspnea in panic disorder. Depression and Anxiety, 20, 32-38.

Rietveld, S., \& Prins, P. J. M. (1998). The relationship between negative emotions and acute subjective and objective symptoms of childhood asthma. Psychological Medicine, 28, 407-415.

Shrout, P. E., \& Fleiss, J. L. (1979). Intraclass correlations: Uses in assessing rater reliability. Psychological Bulletin, 86, 420-428.

Simon, P. M., Schwartzstein, R. M., Weiss, J. W., Lahive, K., Fencl, V., Teghtsoonian, M., et al. (1989). Distinguishable sensations of breathlessness induced in normal volunteers. American Review of Respiratory Disease, 140, 1021-1027.

Skevington, A. M., Pilaar, M., Routh, D., \& Macleod, R. D. (1997). On the language of breathlessness. Psychology and Health, 12, 677-689.

Torgerson, W. S. (1958). Theory and Methods of Scaling. New York: Wiley. Wishart, D. (1982). Clustan user manual. Edinburgh: Program Library Unit, Edinburgh Univ.

\section{Appendix}

List of Respiratory Symptom Descriptors: German Version and English Translation

\begin{tabular}{ll}
\hline \multicolumn{1}{c}{ German descriptors } & \multicolumn{1}{c}{ English descriptors } \\
\hline 1. Meine Atmung erfordert mehr Anstrengung. & My breathing requires more effort. \\
2. Ich fühle mich als würde ich ersticken.* & I feel that I am smothering. \\
3. Ich fühle Hunger nach mehr Luft. & I feel a hunger for more air. \\
4. Ich atme schwer. & My breathing is heavy. \\
5. Ich kann nicht tief Luft holen. & I cannot take a deep breath. \\
6. Ich bin außer Atem. & I feel out of breath. \\
7. Mein Atem geht nicht den ganzen Weg in die Lunge. & My breathing does not go in all the way. \\
8. Mein Brustkorb ist eingeschnürt.* & My chest is constricted. \\
9. Das Atmen erfordert mehr Arbeit. & My breathing requires more work. \\
10. Ich ringe um mehr Luft. & I am panting for more air. \\
11. Mir stockt der Atem.* & I feel that my breath stops. \\
12. Ich schnappe nach Luft. & I am gasping for breath. \\
13. Meine Brust ist eingeengt. & My chest feels tight. \\
14. Mein Atem ist schnell. & I feel that my breathing is rapid. \\
15. Mein Atem ist flach.* & My breathing is shallow. \\
16. Ich habe das Gefühl ich atme mehr. & I feel that I am breathing more. \\
17. Ich bekomme nicht genug Luft. & I cannot get enough air. \\
18. Ich kann nicht genug ausatmen. & My breath does not go out all the way. \\
19. Meine Atmung erfordert mehr Konzentration. & My breathing requires more concentration. \\
20. Mir bleibt die Luft weg.* & I am running out of air. \\
\hline ** Descriptors with an $I C C<.75$ in the German version. &
\end{tabular}

